\title{
Transfer hydrogenation vs nucleophilic addition: aryne reaction with alcohols
}

Keywords: benzyne, nucleophilic addition, transfer hydrogenation

\section{Introduction}

Aryne $^{1}$ intermediates have been well recognized for their soft electrophilic nature that enables them to react with a wide variety of electron-rich substrates, often in a synthetically useful manner. A surprising exception to this reactivity is aliphatic alcohols which, despite their nucleophilic nature, are generally unreactive with arynes. Although several intermolecular examples of benzyne reacting with simple alcohols can be found in the early literature, most of such aryl ether-forming reactions afford products in marginal yields and rely on the use of alcohols as solvents. ${ }^{2-5}$ Recent advances where arynes are generated from the Kobayashi precursor (o-trimethylsilyl aryl triflate $)^{6}$ have only reiterated that stoichiometic quantities of aliphatic alcohols do not undergo nucleophilic addition to arynes in a productive manner. ${ }^{7}$ As an illustrative example, when carbodiimide, a not well recognized nucleophile, and methanol are both present, aryne would selectively react with the former. ${ }^{8}$ This seemingly counterintuitive "inertness" of benzyne toward aliphatic alcohols, along with some others highlighted in our recent review, ${ }^{9}$ constitutes a long-standing curiosity for aryne chemists, both synthetically and mechanistically.

This curiosity has now been addressed computationally by Hoye and co-workers (Scheme 1). ${ }^{10}$ The expected alcohol nucleophilic addition to aryne (Scheme 1, right) was found to be second-order with respect to the alcohol (Figure 1), and consequently is favored only at high concentration of the latter. This computational result is consistent with early success where alcohols were used as solvents, and is also consistent with Hoye's and Lee's earlier results where large excess of alcohols was typically used. ${ }^{11-12}$ Interestingly, this mechanistic rationale is in good agreement with a half-century-old finding from Bunnett that alcohol addition to arynes comes with accumulation of negative charge on aryne. ${ }^{4}$

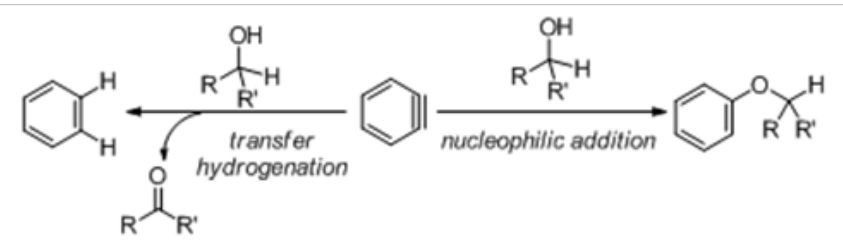

Scheme I Computational mechanism for the second-order nucleophilic addition of $\mathrm{MeOH}$ to benzyne (DFT, M06-2X/6-3II+G (d, p) level with SMD solvation model in $\mathrm{MeOH}$. (Units in $\mathrm{kcal} / \mathrm{mol}$ ).

Additionally, Hoye reported that at low alcohol concentration the reaction can take an alternate path, an Oppenauer- or Cannizzarolike transfer hydrogenation reaction that oxidizes the alcohol into a carbonyl compound and reduces the aryne into an arene (Scheme 1, left). This novel mode of reactivity is first-order with respect to the alcohol and is calculated to proceed via a concerted, asynchronous mechanism (Figure 2). The aryne ring in the transition state is quite distorted $^{13,14}$ and the $\mathbf{H}-\mathbf{C}(\mathrm{OH})$ bond cleavage is more advanced
Volume I Issue I - 2017

\author{
Anton V Dubrovskiy,' Feng Shi² \\ 'Department of Chemistry, University of Houston-Clear Lake, \\ USA \\ ${ }^{2}$ Research Service Division, WuXi AppTec (Wuhan) Company \\ Ltd, China
}

\begin{abstract}
Correspondence: Anton V Dubrovskiy, Department of Chemistry, University of Houston-Clear Lake, Houston, TX, 77058, USA, Tel (28I) 283-3769, Email dubrovskiy@uhcl.edu

Feng Shi, Research Service Division,WuXi AppTec (Wuhan) Company Ltd.666 Gaoxin Road, East Lake High-Tech Development Zone Wuhan 430075, P R China, Emailshi_feng_017677@wuxiapptec.com
\end{abstract}

Received: February 28, 2017| Published: April 12, 2017

than the cleavage of the $\mathrm{O}-\mathrm{H}$ bond. Both findings support a minor contribution of a hydridic nature of the transferred hydrogen $(\mathbf{H}$ at the carbinol carbon atom) within the concerted mechanism frame (a real carbocation mechanism is excluded). Due to its kinetic profile, the transfer hydrogenation is favored at low alcohol concentrations (Figure 3A). Supportive of the proposed mechanism, this process was also shown to be favored for substrates with weaker $\mathbf{H}-\mathbf{C}(\mathrm{OH})$ bonds (Figure 3B).

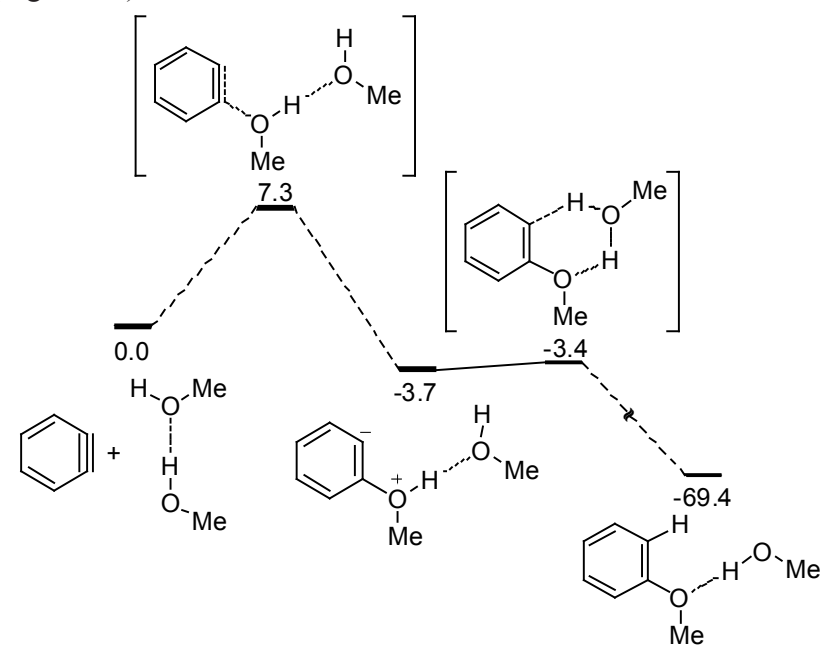

Figure I Computational mechanism for the second-order nucleophilic addition of $\mathrm{MeOH}$ to benzyne (DFT, M06-2X/6-3II+G (d, p) level with SMD solvation model in $\mathrm{MeOH}$. Units in $\mathrm{kcal} / \mathrm{mol})$.

This previously unrecognized transfer hydrogenation and equally unrecognized second-order alcohol addition have collectively explained the poor reactivity in the addition of alcohols to arynes. Meanwhile, it also sparked another curiosity standing equally elusive, that is, whether the lack of reactivity of aliphatic carboxylic acids and primary carboxyamides with arynes ${ }^{7}$ could be explained by the same model. In fact, if one compares Hoye's and Lee's results ${ }^{11,12}$ where 
acetic acid underwent nucleophilic addition to arynes with Larock's conditions ${ }^{7}$ where no synthetically meaningful yield was obtained, it follows that the former success can be, at least partially, attributed to the excess and higher concentration of $\mathrm{AcOH}^{15}$ which in turn might suggest higher-order kinetics with respect to the acid. It remains questionable as to why phenols behave differently from aliphatic alcohols in the nucleophilic addition with arynes, and why aromatic carboxylic acids behave differently from aliphatic ones.

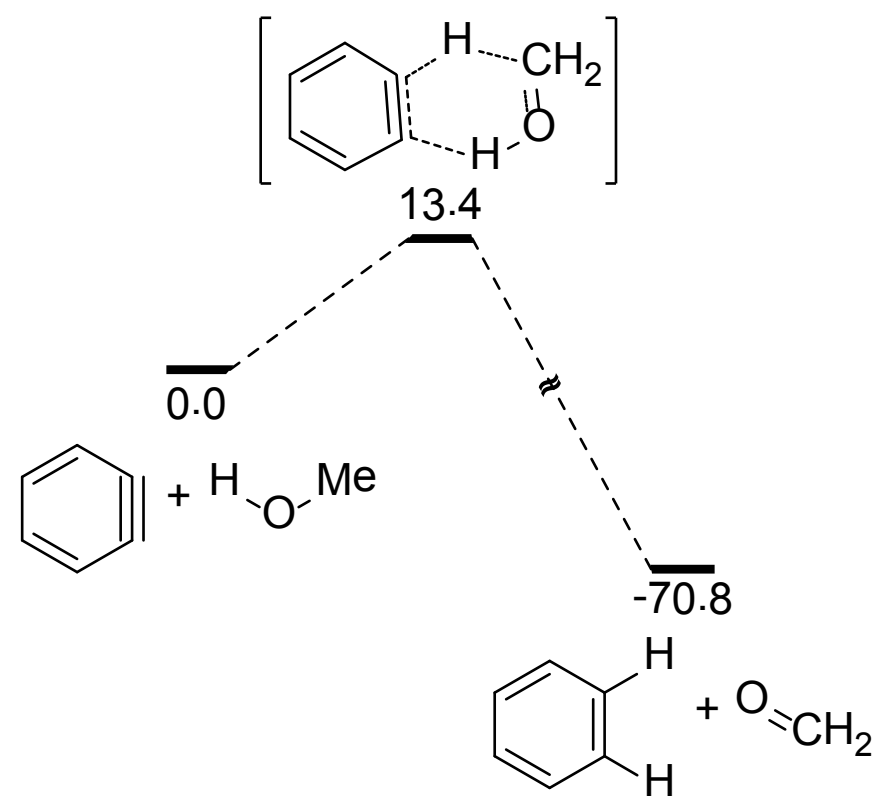

Figure 2 Computational mechanism for the concerted transfer hydrogenation of $\mathrm{MeOH}$ to benzyne (DFT, M06-2X/6-3II+G(d,p) level in gas phase. Units in $\mathrm{kcal} / \mathrm{mol})$.

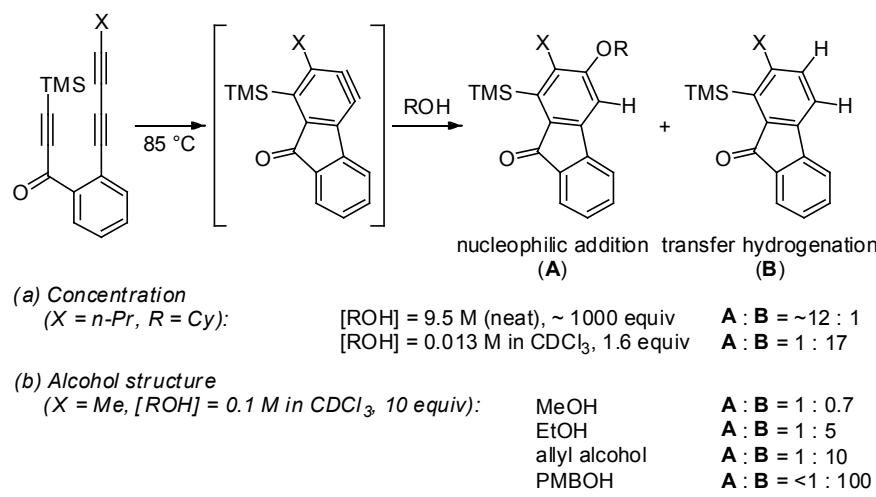

Figure 3 Nucleophilic addition vs transfer hydrogenation: dependence on the concentration and structure of alcohol $(\mathrm{Cy}=$ cyclohexyl, $\mathrm{PMB}=$ 4-methoxylbenzyl).

The transfer hydrogenation reactivity may also be more general than imagined. Hoyle reported that other than alcohols, medium-sized cycloalkanes and even THF are good hydrogen donors to reduce arynes. ${ }^{16}$ Okuma also reported ${ }^{17}$ that $\alpha$-amino acid methyl esters derived from phenylalanine and aspartic acid resulted in significant dehydrogenation (product E, Figure 4) after annulation to indolin-3ones. Because tyrosine counterpart did not afford the dehydrogenation product, this process is unlikely aerobic. Therefore, a transfer hydrogenation (Figure 4) is possibly in operation. This and related dehydrogenation cases need to be addressed in the future work.

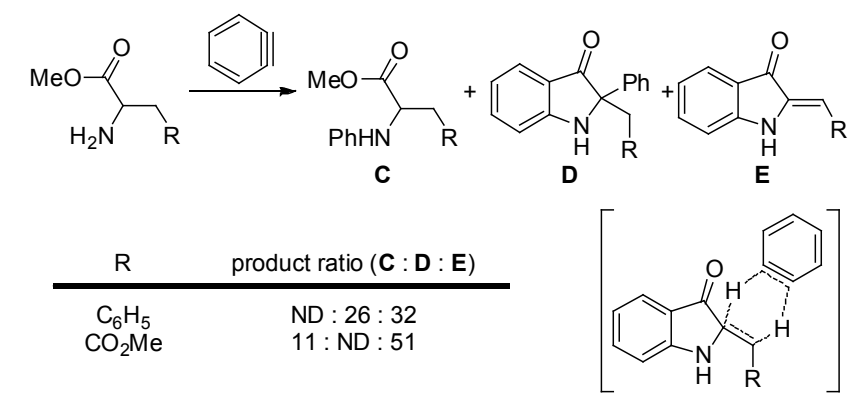

Figure 4 Dehydrogenation of some indolin-3-ones and a possibility of aryne serving as the oxidant.

\section{Conclusion}

In summary, the work of Hoye and co-workers shed light on previously unexplained reactivity of arynes with alcohols. Well supported by experimental evidence, this computational study not only explains the apparent inertness of alcohols toward arynes, but also provides solid evidence for a novel transfer hydrogenation process that can be in prospect applied to a broader scope of aryneparticipated redox schemes. It is a good demonstration of how diligent studies of seemingly well understood reagents/reactions can unfold new and unexpected modes of reactivity.

\section{Acknowledgements}

This work was partially supported by the Robert Welch Foundation (departmental grant BC-0022).

\section{Conflict of interest}

The author declares no conflict of interest.

\section{References}

1. Referring herein to ortho-benzynes and their derivatives or heterocyclic analogues.

2. Stiles M, Miller RG, Burckhardt U. For benzyne generated from diazotized anthranilic acid reacting with $t-B u O H$. J Am Chem Soc. 1963;85:1792-1797.

3. Wittig G, Hoffmann RW. For benzyne generated from benzo[d] $[1,2,3]$ thiadiazole 1, 1-dioxide reacting with mixed water and $\mathrm{EtOH}$ (forming phenol and phenetole). Angew Chem. 1961;73:435-436.

4. Bunnett JF, Happer DAR, Patsch M, et al. For benzynes generated from diazotized anthranilic acid and 1-(o-haloaryl)-2-benzenesulfonhydrazide reacting with $\mathrm{MeOH}$. Am Chem Soc. 1966;88:5250-5254.

5. Ikawa $T$, Nishiyama $T$, Shigeta $T$, et al. For one example where an aryne generated from the Kobayashi precursor reacting with excess EtOH in THF $([\mathrm{EtOH}]=0.21 \mathrm{M}$, also with a high water concentration from TBAF), Angew. Chem Int Ed. 2011;50:5674-5677.

6. Himeshima Y, Sonoda T, Kobayashi H. Fluoride-induced 1,2-elimination of $o$-trimethylsilylphenyl triflate to benzyne under mild conditions. Chem Lett. 1983;12(8):1211-1214.

7. Liu Z, Larock RC. Facile $N$-Arylation of Amines and Sulfonamides and $O$-Arylation of Phenols and Arenecarboxylic Acids. J Org Chem. 2006;71(8):3198-3209.

8. Li R, Tang H, Fu H, et al. Arynes double bond insertion/nucleophilic addition with vinylogous amides and carbodiimides. J Org Chem. 2014;79(3):1344-1355. 
9. Wu C, Shi F. A Closer Look at Aryne Chemistry:Details that Remain Mysterious. Asian J Org Chem. 2013;2(2):116-125.

10. Willoughby PH, Niu D, Wang T, et al. Mechanism of the Reactions of Alcohols with $O$-Benzynes. J Am Chem Soc. 2014;136(39):1365713667.

11. Hoye TR, Baire B, Niu D, et al. The hexadehydro-Diels-Alder Reaction. Nature. 2012;490(7419):208-212.

12. R Karmakar, SY Yun, KP Wang, et al. Regioselectivity in the nucleophile trapping of arynes: the electronic and steric effects of nucleophiles and substituents. Org Lett. 2014;16(1):6-9.

13. Cheong PHY, Paton RS, Bronner SM, et al. Indolyne and Aryne Distortions and Nucleophilic Regioselectivites. $J$ Am Chem Soc. 2010;132:1267-1269.
14. Yoon JIG, Bronner SM, Goetz AE, et al. Indolyne Experimental and Computational Studies:Synthetic Applications and Origins of Selectivities of Nucleophilic Additions. J Am Chem Soc. 2010;132(50):17933-17944

15. The actual situation may be even more dramatic. As the concentrations of aryne precursors are far more than those of free arynes, the effective equivalents of $\mathrm{AcOH}$, or other nucleophiles, to free arynes is far more than the amount added. The exact magnitude may depend on the rate of aryne generation from a certain precursor.

16. Niu D, Willoughby PH, Woods BP, et al. Alkane desaturation by concerted double hydrogen atom transfer to benzyne. Nature. 2013;501:531-534.

17. Okuma K, Matsunaga N, Nagahora N, et al. Chem Commun. 2011;47:5822-5824. 